\title{
Transequatorial Connections: Loops or Magnetic Separators?
}

\author{
Alexei A. Pevtsov ${ }^{1}$ \\ ${ }^{1}$ National Solar Observatory†, Sunspot, NM 88349, USA \\ email: apevtsov@nso.edu
}

\begin{abstract}
.
We provide a brief overview of properties of transequatorial loops (TLs) and show that some TLs fit the description of a loop, while others appear to be the magnetic separators.
\end{abstract}

\section{Introduction}

Transequatorial loops (TLs) may be relevant to three major solar phenomena: dynamo, reconnection, and eruption.

(a) Babcock (1961) proposed that the connectivity of active regions across the solar equator is necessary to convert the toroidal flux to the poloidal flux.

(b) Some transequatorial connections may represent the best examples of the magnetic reconnection (e.g., Tsuneta 1996).

(c) Because of their North-South orientation, the erupting TLs may have greater geomagnetic effects than the regular active region loops (Khan \& Hudson 2000, Glover et al 2003).

The aim of this article is to provide a brief overview of TLs' properties. Earlier studies of TLs can be found in Van Speybroeck, Krieger \& Vaiana (1970), Chase et al. (1976), Fárník, Karlický \& Švestka (1999), and Pevtsov (2000).

\section{General Statistics}

We extend our previous survey of transequatorial structures (Pevtsov 2000) using the observations from the Yohkoh's soft X-ray Telescope (SXT, Tsuneta et al. 1991). We define a TL as a system of bright coronal features connecting two distinct areas across the solar equator. Some TLs appear as a single loop, other may be comprised of multiple loop-like features. In the latter case, we call the entire system a single TL even if individual loops may be seen in different times. We disregard the fact that TLs may appear and disappear as a corresponding area crosses the solar disk. In a framework of this definition, we have identified 226 TLs between 1991 October - 2001 November.

TLs included in the dataset vary in length from about $15-75^{\circ}$; the mean length is about $30^{\circ}$ (Figure 1a). Although the number of TLs varies with sunspot cycle (Figure 1b), the fraction of active regions showing transequatorial loops does not significantly change between the solar minimum and maximum (Figure 1d). Overall about $35 \%$ of all active regions exhibit the transequatorial connections.

Figure 1c shows tilt of TLs defined as an angle between the solar equator and the line connecting TL's footpoints. When the northern footpoint is situated eastward from the southern footpoint, the tilt is $>90^{\circ}$. The average tilt exhibits a systematic asymmetry,

$\dagger$ Operated by AURA, Inc under cooperative agreement with the National Science Foundation 

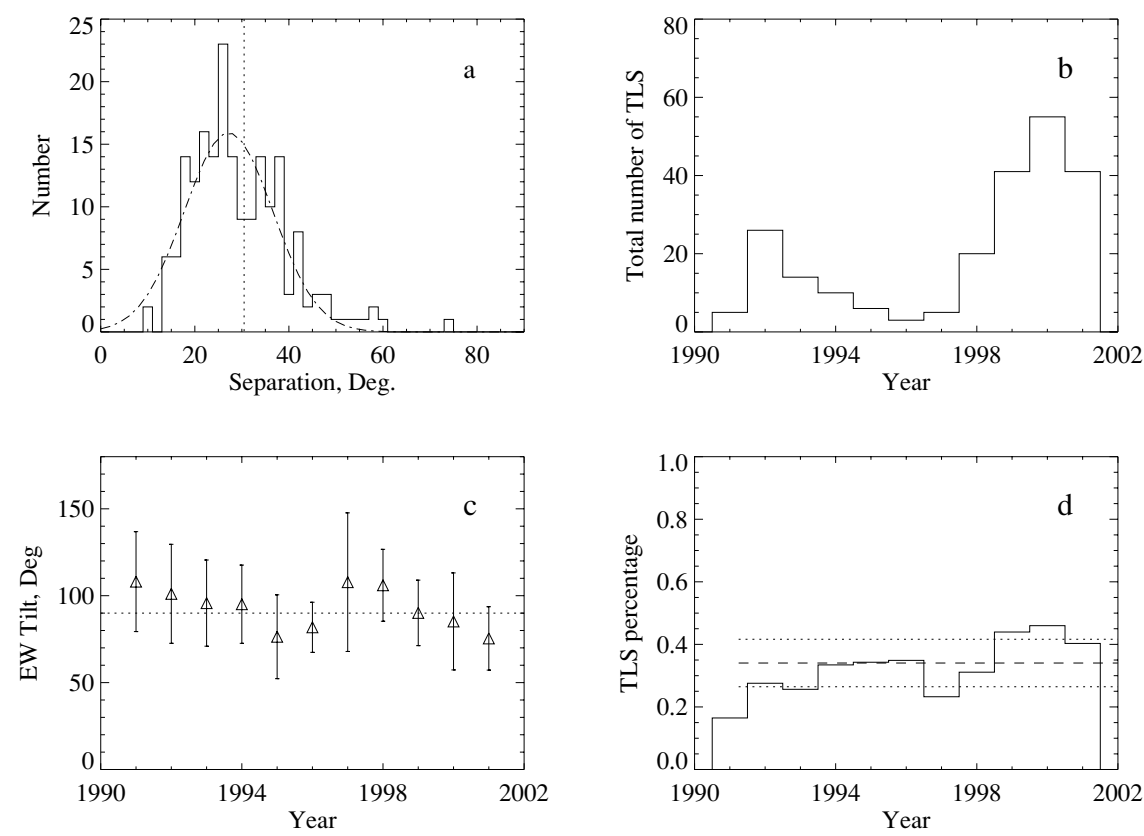

Figure 1. (a) - Length (footpoint separation) of 226 TLs; stippled line is a Gaussian fit, vertical dotted line shows mean of the distribution, (b) - Annual number of TLs, (c) - East-West tilt of TLs; dotted line shows average tilt, (d) - number of TLs normalized by the annual sunspot number; dashed and dotted lines show the average and \pm one standard deviation.

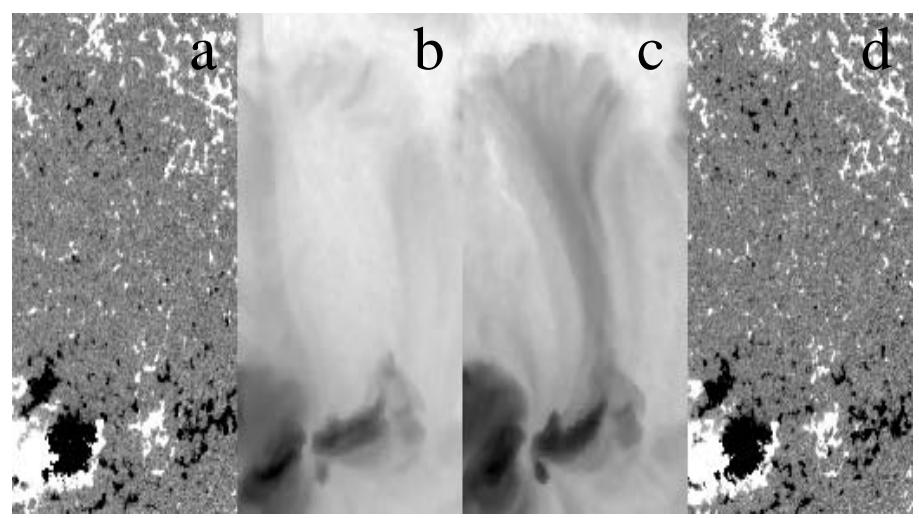

Figure 2. Brightening of pre-existing transequatorial connections (a) - MDI (17-MAY-00 20:48:03 UT), (b) - SXT (Al.1, 17-MAY-00 21:33:23 UT), (c) - SXT (Al.1, 18-MAY-00 02:52:03 UT), (d) - MDI (18-MAY-00 03:12:03 UT).

which suggests that the transequatorial connections are not the result of a random encounter of two close regions. Previous results (Chase et al. 1976, Pevtsov 2000) support that the TLs represent magnetic connectivity within activity complexes. For example, a majority of TLs develops between two magnetic areas of alike helicity. The longitudinal distribution of TLs (Figure 6 in Pevtsov 2000) shows that the TLs develop only in particular longitudes, while the active regions are formed at all longitudes. In some cases, TLs between two areas on the Sun may exist prior to the emergence of active regions. 

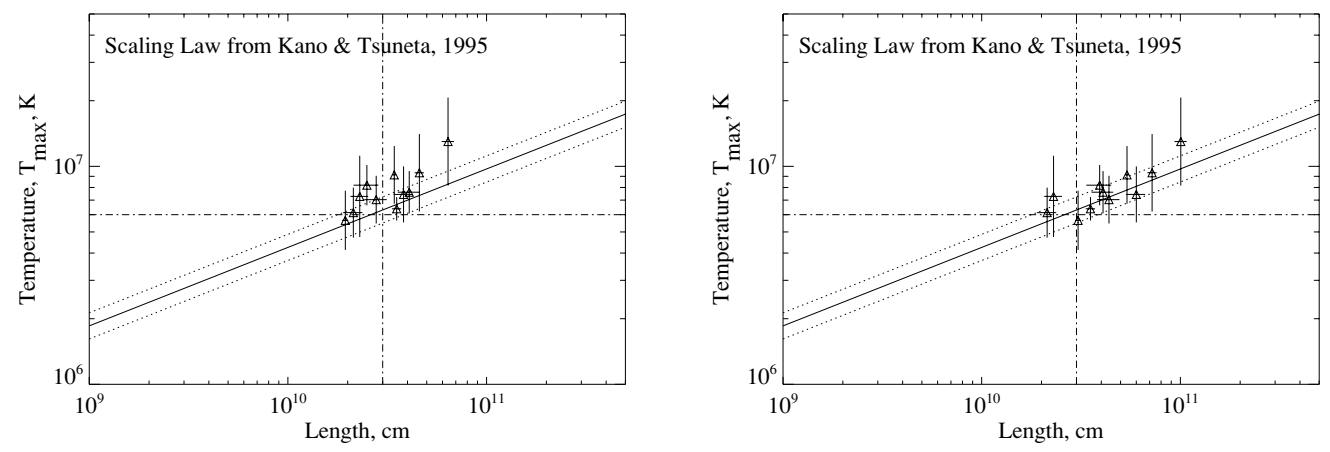

Figure 3. TLs temperature vs. (left panel) observed and (right) corrected length. Solid and dotted lines show scaling law and its standard deviation from Kano \& Tsuneta (1995). Dash-dotted lines mark maximuma for the AR loops included in Kano \& Tsuneta's data set.

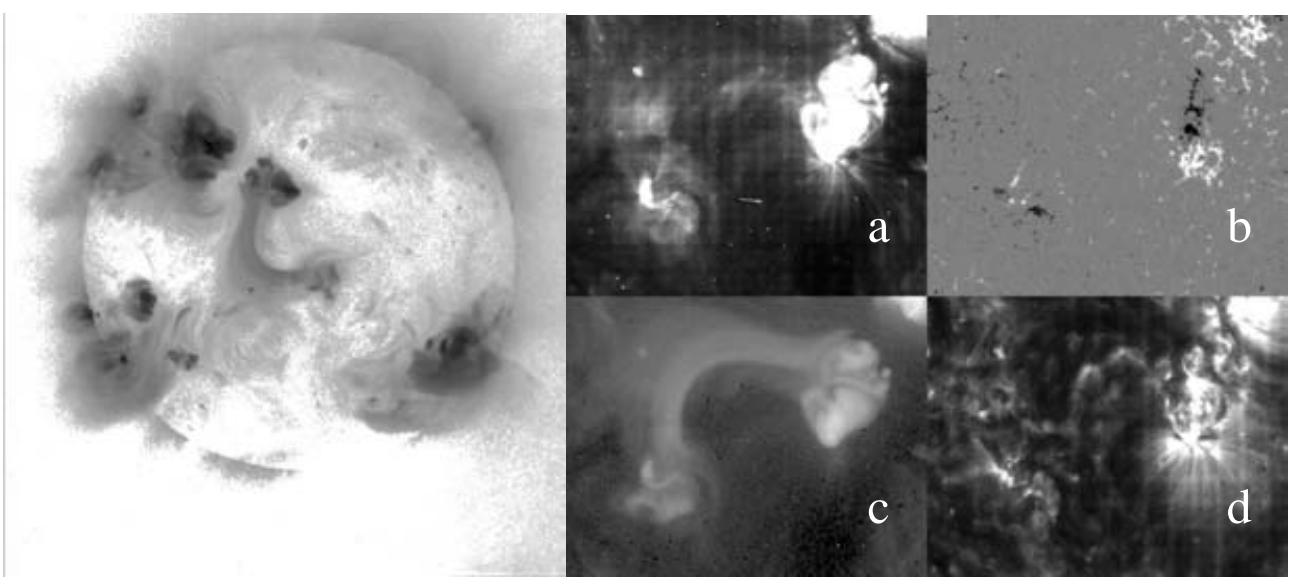

Figure 4. Left panel: TL observed by SXT/Yohkoh, Right panel: same TL (rotated) in

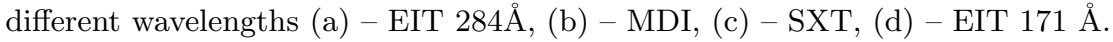

Pevtsov (2000, see Figure 4) has shown that TLs between two active regions may develop shortly after the emergence of a second region. On the other hand, some TLs may appear without significant changes in the photospheric magnetic field (Figure 2). Such evolution implies that the transequatorial connection may exist prior to the appearance of a bright loop-like structure in the corona as suggested by Švestka et al. (1977).

Figure 3 shows the temperature of selected TLs versus their length. A crude estimate of the maximum height of TLs (made using a change in projection in 12 hours) suggests that some TLs are low laying structures. Other TLs may be best described as semicircular loops. Using the observed length as a proxy for the total length (Figure 3, left), it shows that the majority of TLs deviate from the loops' scaling law established by Kano \& Tsuneta (1995). Correcting the loops' lengths under the assumption of a semicircular shape improves the agreement (Figure 3, right) although some loops still deviate significantly from the scaling law. Given the uncertainty in the true length, we consider Figure 3 inconclusive although in both cases there are structures that do not fit the loops' scaling law.

Figure 4 shows an example of a TL, which properties cannot be understood in a framework of a loop model. The TL rises high in the corona, but it does not expand with 

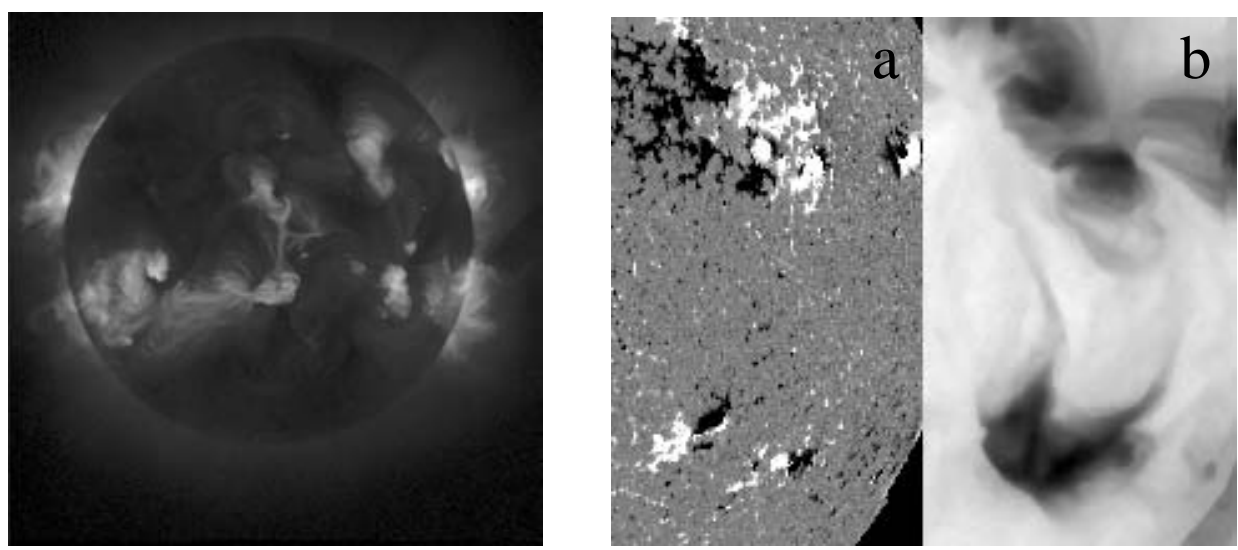

Figure 5. Examples of transequatorial structures that exhibit properties of current sheets.

the height. There is a gap between the TL footpoints and the AR loop system below. The TL does not extend to the photospheric magnetic poles. It ends near the top of the AR loop-system and continues around it toward the photosphere. By its appearance, the structure is reminiscent of a 3-D reconnection model (Priest and Schrijver, 1999) with the TL been a spine and the TL footpoint structure been a fan surface. The magnetic structure of this TL cannot be modelled as a current-free field (Longcope 2004, private communication).

Figure 5 shows other examples of TLs that cannot be described in a framework of a loop model.

\section{Conclusions}

(a) Transequatorial connections are present at any phase of the solar cycle. The TLs number (TLN) varies with the sunspot number (SPN). The TLN/SPN ratio remains constant within 1- $\sigma$ over the solar cycle.

(b) TLs are best seen in broadband SXT data although they can also be identified in EIT $284 \AA$ observations (Figure 4).

(c) Asymmetry in tilt of TLs, a preference for alike helicity regions to show TLs, and non-uniform longitudinal distribution of TLs suggest that the transequatorial connections develop within complexes of activity.

(d) Some TLs fit the interpretation of a coronal loop, but many appear to be the magnetic separators or the current sheets.

\section{References}

Babcock, H.W. 1961 Astrophys. J. 304, 542-559.

Chase, R.C., Krieger, A.S., Švestka, Z. \& Vaiana, G.S. 1976 Space Research XVI, 917-922.

Fárník, F., Karlický, M. \& Švestka, Z. 1999 Solar Phys. 187, 33-44.

Glover, A., Harra, L. K., Matthews, S. A. \& Foley, C. A. 2003 Astron. Astrophys. 400, 759-767.

Kano, R. \& Tsuneta, S. 1995 Astrophys. J. 454, 934-944.

Khan, J. I. \& Hudson, H. S. 2000 Geophys. Res. Lett. 27, 1083-1086.

Pevtsov, A.A. 2000 Astrophys. J. 531, 553-560.

Priest, E. R. \& Schrijver, C. J. 1999 Solar Phys. 190, 1-24.

Švestka, Z., Krieger, A.S., Chase, R.C., \& Howard, R. 1977 Solar Phys. 52, 69-90.

Tsuneta, S. et al. 1991 Solar Phys. 136, 37-67.

Tsuneta, S. 1996 Astrophys. J. 456, L63-L65.

Van Speybroeck, L.P., Krieger, A.S. \& Vaiana, G.S. 1970 Nature 227, 818-822. 